\title{
Energy and electronic characteristics of silicon polyprismanes: density functional theory study
}

\author{
M. A. Gimaldinova ${ }^{1}$, K. P. Katin ${ }^{1,2, \dagger}$, M. A. Salem ${ }^{1,3}$, M. M. Maslov ${ }^{1,2}$ \\ †KPKatin@yandex.ru \\ ${ }^{1}$ National Research Nuclear University “MEPhI”, 31 Kashirskoe Shosse, Moscow, 115409, Russia \\ ${ }^{2}$ Research Institute for the Development of Scientific and Educational Potential of Youth, \\ 14/55 Aviatorov str., Moscow, 119620, Russia \\ ${ }^{3}$ Department of Physics, Faculty of Science, Zagazig University, Zagazig, 44519, Egypt
}

\begin{abstract}
We report structural, energetic and some electronic properties of $[n, 4]-,[n, 5]-$, and $[n, 6]$ silaprismanes (polysilaprismanes), i.e. silicon nanotubes of a special type constructed from dehydrogenated molecules of cyclosilanes (silicon rings) $\mathrm{Si}_{4}^{-}$, $\mathrm{Si}_{5}{ }^{-}$, and $\mathrm{Si}_{6}$, respectively. For large $n$, polysilaprismanes can be considered as the analogs of silicon nanotubes with an extremely small cross-section in the form of a regular polygon. Binding energies, interatomic bonds, and the energy gaps between the highest and lowest occupied molecular orbitals (HOMO and LOMO, respectively) have been calculated using the density functional theory for the systems up to ten layers. It is found that $[n, 4]$ silaprismane is not thermodynamically stable in the bulk limit $(n \rightarrow \infty)$, while the $[n, 5]$ - and $[n, 6]$ silaprismanes conserve their highly strained framework and become more thermodynamically stable as the number of layers $n$ increases. Moreover, the HOMO-LUMO gap analysis reveals that the $[n, 5]$ - and [ $n, 6]$ silaprismanes with the large effective length can be referred to semimetals or even the conductors. So, they can be successfully used unlike the carbon analogs in nanoelectronics as functional nanowires or the basis for the computational logic elements without any additional doping or applying the mechanical stresses. Thicknesses of silaprismanes are comparable with that of the smallest carbon nanotubes.
\end{abstract}

Keywords: silicon polyprismanes, density functional theory, geometry, energy and electronic characteristics.

\section{Introduction}

Well-known carbon $[n, m]$ prismanes (polyprismanes) can be regarded as stacked layers of dehydrogenated cycloalkane molecules, where $m$ is the number of vertices of the closed ring and $n$ is the number of layers. Carbon prismanes are of significant fundamental and applied interest [ $1-5]$. They belong to the family of tubular quasi-1D $s p^{3}$-hybridized carbon materials [6-7]. Some novel carbon materials with attractive mechanical characteristics contain prismanelike structural fragments [8-10]. Intensive theoretical and experimental studies of polyprismanes were already carried out. It is established that the thermodynamic stability of such systems increases and the HOMO-LUMO gap decreases with the increasing number of layers. However, it is found that the kinetic stability of such carbon nanostructures decreases with increasing number of layers in the system. At the same time, it is known that the silicon nanostructures can be used in various electronic, optoelectronic, thermoelectric, and biological applications [11]. Silicon, as well as carbon, belongs to the fourth group of elements. Therefore, the silicon atoms have the carbon-like electronic configuration. Previously, different systems based on silicon were studied: silicon nanoparticles [12], silicon clusters [13-16], silicon nanowires [17-20], silicon tubular structures [21, 22], etc. Thus, the idea of replacing carbon with silicon in polyprismanes has been partially developed. In the presented study we introduce silicon $[n, m]$ prismanes or silaprismanes. They represent silicon atomic rings stacked on the top of each other by layers similarly to carbon polyprismanes (Fig. 1). Note that some of such small structures are already successfully synthesized $[23,24]$.

In this paper, we calculate the ground-state properties of silicon polyprismanes at zero temperature and pressure. We have performed structural optimization of $[n, m]$ silaprismanes, and also estimated their electronic, energy, and geometric characteristics using the density functional theory. We have analyzed the behavior of these properties in the bulk limit $(n \rightarrow \infty)$.

\section{Materials and methods}

In our study density functional theory (DFT) with Becke's three-parameter hybrid method and the Lee-Yang-Parr exchange-correlation energy functional (B3LYP) $[25,26]$ with the electron basis set of $6-311 \mathrm{G}(\mathrm{d}, \mathrm{p})$ [27] were used to optimize the geometries and obtain the structural, energy and electronic (namely HOMO-LUMO gaps) characteristics of the silicon polyprismanes. The density functional theory (DFT) has been shown to be a basic method for the analysis of the properties of low-dimensional nanostructures with highly strained frameworks [28-31] and has been successful in terms of computing speed and accuracy [32]. All DFT calculations were performed using the TeraChem program 
package [33-36]. During the geometry optimization, the global charge of all systems considered was neutral. The maximum force and root mean square forces were $4.5 \times 10^{-4}$ and $3 \times 10^{-4}$ (hartrees/bohr and hartrees/radian), whereas the maximum displacement and root mean square displacement were $1.8 \times 10^{-3}$ and $1.2 \times 10^{-3}$. The samples studied are silicon $[n, m]$ prismanes with $m=4,5$, and 6 (silicon tetra-, penta-, and hexaprismanes, respectively), and $n=2 \div 10$ (see Fig. 1). Note that for all polyprismanes studied we also calculated the frequency spectra in the framework of the same level of theory after the geometry optimization. All frequencies were positive for each metastable configuration.

\section{Results and discussion}

\subsection{Geometry characteristics}

We distinguished two types of Si-Si bonds for silaprismanes: parallel to the silaprismane main axis interlayer $\mathrm{Si}-\mathrm{Si}$ bonds $\left(l_{\|}\right)$and perpendicular to the main axis intralayer $\mathrm{Si}-\mathrm{Si}$ bonds $\left(l_{\perp}\right)$. The obtained values for the considered systems are presented in Table 1 . It can be seen from Table 1 that in the case of $m=4$ the values of $l_{\|}$and $l_{\perp}$ grow steadily with the increasing number of the layers. In addition, it was found that $[10,4]$ silaprismane was not thermodynamically stable.

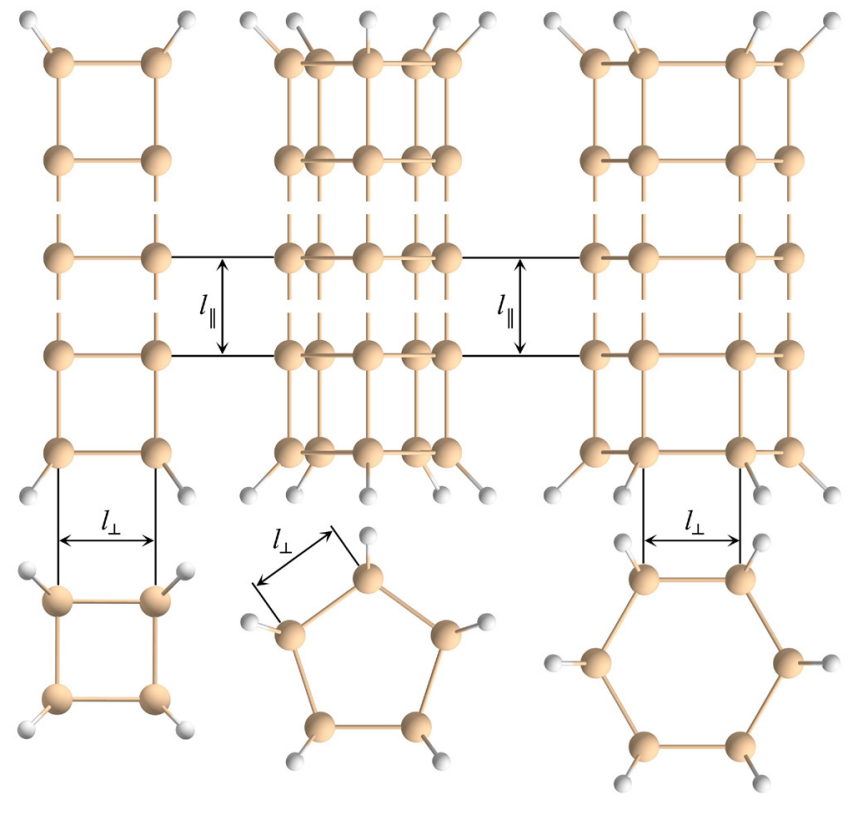

a

C

Fig. 1. Molecular structures of $[n, 4]-(\mathrm{a}),[n, 5]-(\mathrm{b})$ and $[n, 6]$ silaprismanes (c). Top-side view and bottom-top view. Symbols $l_{\|}$and $l \perp$ correspond to the interlayer and intralayer Si-Si bonds, respectively.

Table 1. Bond lengths of $[n, m]$ silaprismanes $(m=4,5$, and 6) obtained in the frame of DFT/B3LYP/6-311G(d,p) level of theory: $l_{\|}$and $l_{\perp}$ - interlayer and intralayer Si-Si bonds, respectively, $l_{\mathrm{Si}-\mathrm{H}}-\mathrm{Si}-\mathrm{H}$ bond lengths, $\bar{l}-$ corresponding averaged bond lengths. All bond lengths are quoted in angstroms.

\begin{tabular}{|c|c|c|c|c|c|c|c|}
\hline$m$ & $n$ & $l_{\|}$ & $\bar{l}_{\|}$ & $l_{\perp}$ & $\bar{l}_{\perp}$ & $l_{\mathrm{Si}-\mathrm{H}}$ & $\bar{l}_{\mathrm{Si-H}}$ \\
\hline \multirow{8}{*}{4} & 2 & 2.4 & 2.4 & 2.401 & 2.401 & 1.491 & 1.491 \\
\hline & 3 & 2.394 & 2.394 & $2.382 \div 2.439$ & 2.42 & 1.496 & 1.496 \\
\hline & 4 & $2.411 \div 2.424$ & 2.415 & $2.41 \div 2.434$ & 2.422 & 1.494 & 1.494 \\
\hline & 5 & $2.411 \div 2.426$ & 2.416 & $2.42 \div 2.449$ & 2.426 & 1.493 & 1.493 \\
\hline & 6 & $2.409 \div 2.427$ & 2.419 & $2.406 \div 2.459$ & 2.43 & 1.493 & 1.493 \\
\hline & 7 & $2.409 \div 2.429$ & 2.419 & $2.391 \div 2.493$ & 2.434 & 1.492 & 1.492 \\
\hline & 8 & $2.407 \div 2.428$ & 2.419 & $2.398 \div 2.485$ & 2.434 & $1.492 \div 1.493$ & 1.492 \\
\hline & 9 & $2.411 \div 2.428$ & 2.42 & $2.391 \div 2.496$ & 2.436 & $1.492 \div 1.493$ & 1.492 \\
\hline \multirow{9}{*}{5} & 2 & 2.397 & 2.397 & $2.392 \div 2.393$ & 2.393 & 1.493 & 1.493 \\
\hline & 3 & 2.396 & 2.396 & $2.351 \div 2.436$ & 2.408 & 1.496 & 1.496 \\
\hline & 4 & $2.413 \div 2.43$ & 2.419 & $2.388 \div 2.428$ & 2.409 & $1.494 \div 1.495$ & 1.495 \\
\hline & 5 & $2.409 \div 2.429$ & 2.419 & 2.397 & 2.414 & 1.495 & 1.495 \\
\hline & 6 & $2.412 \div 2.434$ & 2.424 & $2.385 \div 2.445$ & 2.419 & 1.496 & 1.496 \\
\hline & 7 & $2.412 \div 2.434$ & 2.427 & $2.393 \div 2.452$ & 2.421 & $1.495 \div 1.496$ & 1.495 \\
\hline & 8 & $2.41 \div 2.432$ & 2.425 & $2.391 \div 2.439$ & 2.423 & 1.495 & 1.495 \\
\hline & 9 & $2.409 \div 2.429$ & 2.424 & $2.388 \div 2.448$ & 2.424 & $1.494 \div 1.495$ & 1.495 \\
\hline & 10 & $2.431 \div 2.409$ & 2.425 & $2.39 \div 2.446$ & 2.425 & 1.495 & 1.495 \\
\hline \multirow{9}{*}{6} & 2 & $2.392 \div 2.392$ & 2.392 & 2.395 & 2.395 & 1.493 & 1.493 \\
\hline & 3 & $2.39 \div 2.391$ & 2.391 & $2.348 \div 2.443$ & 2.411 & 1.497 & 1.497 \\
\hline & 4 & $2.407 \div 2.426$ & 2.414 & $2.389 \div 2.432$ & 2.411 & 1.496 & 1.496 \\
\hline & 5 & $2.405 \div 2.426$ & 2.416 & $2.398 \div 2.438$ & 2.415 & 1.496 & 1.496 \\
\hline & 6 & $2.404 \div 2.427$ & 2.417 & $2.38 \div 2.447$ & 2.418 & 1.496 & 1.496 \\
\hline & 7 & $2.407 \div 2.429$ & 2.421 & $2.388 \div 2.455$ & 2.419 & 1.496 & 1.496 \\
\hline & 8 & $2.406 \div 2.428$ & 2.421 & $2.387 \div 2.437$ & 2.42 & 1.496 & 1.496 \\
\hline & 9 & $2.406 \div 2.429$ & 2.423 & $2.385 \div 2.445$ & 2.421 & 1.496 & 1.496 \\
\hline & 10 & $2.405 \div 2.427$ & 2.421 & $2.386 \div 2.442$ & 2.422 & $1.495 \div 1.496$ & 1.496 \\
\hline
\end{tabular}


Its highly strained silicon framework decomposes during the structural optimization. Thus, it is not taken into account for the further analysis.

The ranges of bond lengths for long silaprismanes are caused by the edge effects including $\mathrm{Si}-\mathrm{H}$ interactions. Note that the value of $\bar{l}_{\perp}$ increases with the number of layers for every $m(4,5$, or 6$)$.

\subsection{Size dependence of the binding energies and HOMO-LUMO gaps}

The binding energies of $[n, m]$ silaprismanes $\mathrm{Si}_{l} \mathrm{H}_{k}$ are calculated by the equation

$$
E_{b}\left[\frac{\mathrm{eV}}{\text { atom }}\right]=\frac{1}{N_{a t}}\left[k E(\mathrm{H})+l E(\mathrm{Si})-E_{t o t}(\text { polysilaprismane })\right],
$$

where $N_{a t}=k+l$ is the total number of atoms in the polysilaprismane, $E(\mathrm{Si})$ and $E(\mathrm{H})$ are the energies of isolated silicon and hydrogen atoms, respectively, and $E_{\text {tot }}$ (polysilaprismane) is the total energy of the corresponding polyprismane. The polysilaprismane with higher binding energy $E_{b}$ (lower potential energy) is more thermodynamically stable and vice versa. HOMO-LUMO gap $\Delta_{\mathrm{HL}}$ is defined as the energy gap between the highest occupied molecular orbital and the lowest unoccupied molecular orbital. The binding energies and the HOMO-LUMO gaps obtained for $[n, m]$ silaprismanes are summarized in Figs. 2 and 3, respectively.

For $[n, m]$ silaprismanes, we found that $E_{b}$ depends linearly on $n^{-1}$ (here we do not take into consideration point with $n=2)$. The corresponding analytical forms are

$$
\begin{array}{ll}
m=5: & E_{b}=3.16-0.34 n^{-1}, \\
m=6: & E_{b}=3.15-0.32 n^{-1} .
\end{array}
$$

As evident from Fig. 2, the binding energy increases for all considered systems as the number of layers grows, reaching

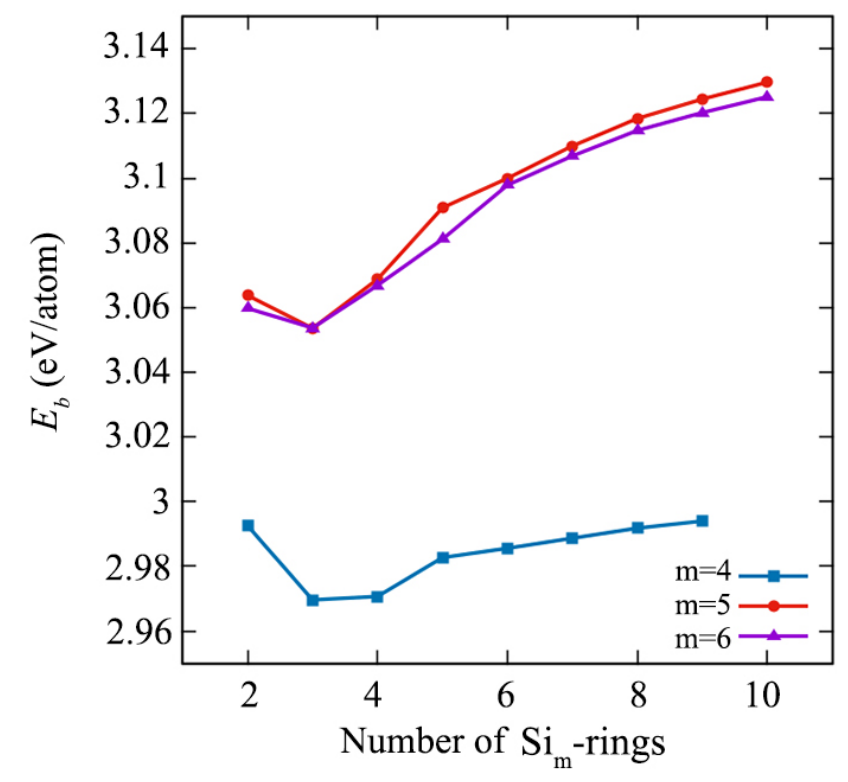

Fig. 2. Binding energies $E_{b}$ versus the number of $\mathrm{Si}_{m}$-rings $n$ obtained at the DFT/B3LYP/6-311G(d,p) level of theory $(m=4,5$, and 6). the following values for "infinite" polysilaprismanes: $E_{b}(\infty)=3.16 \mathrm{eV} /$ atom for $m=5$ and $E_{b}(\infty)=3.15 \mathrm{eV} /$ atom for $m=6$.

HOMO-LUMO gaps $\Delta_{\mathrm{HL}}$ also depend linearly on $n^{-1}$. The corresponding analytical forms are

$$
\begin{array}{ll}
m=5: & \Delta_{\mathrm{HL}}=0.17+5.96 n^{-1}, \\
m=6: & \Delta_{\mathrm{HL}}=0.15+6.33 n^{-1} .
\end{array}
$$

As evident from Fig. 3, the HOMO-LUMO gap decreases for all considered systems as the number of layers grows, reaching the following values for "infinite" polysilaprismanes: $\Delta_{\mathrm{HL}}(\infty)=0.17 \mathrm{eV}$ or $m=5$ and $\Delta_{\mathrm{HL}}(\infty)=0.15 \mathrm{eV}$ for $m=6$. It should be noted that these values are much lower than the corresponding values for the analogs carbon polyprismanes in the "infinite" approximation. For example, for carbon $[n, 5]$ prismanes $\Delta_{\mathrm{HL}}(\infty)=3.2 \mathrm{eV}[5]$.

In general, the increase of binding energy indicates that $[n, 5]$ - and $[n, 6]$ silaprismanes become more thermodynamically stable as the number of layers $n$ increase. High thermodynamic stability of "long" polysilaprismanes can be associated with the decreasing of boundary induced structural distortions as the number of layers increases. As for the HOMO-LUMO gap, such behavior suggests that these nanostructures can be classified as conductors. In the macroscopic limit, the $\Delta_{\mathrm{HL}}$ values allow electronic conductivity in polysilaprismanes without any additional doping or mechanical stresses.

\section{Conclusions}

In this paper, we presented structural, energy, and some electronic properties of $[n, 4]-,[n, 5]-$, and $[n, 6]$ silaprismanes with $n=2 \div 10$. The data of computer modeling obtained in this study indicate an increase of thermodynamic stability of polysilaprismanes with $m=5$ and 6 as the number of layers $n$ increases. Unfortunately, $[n, 4]$ silaprismanes turn

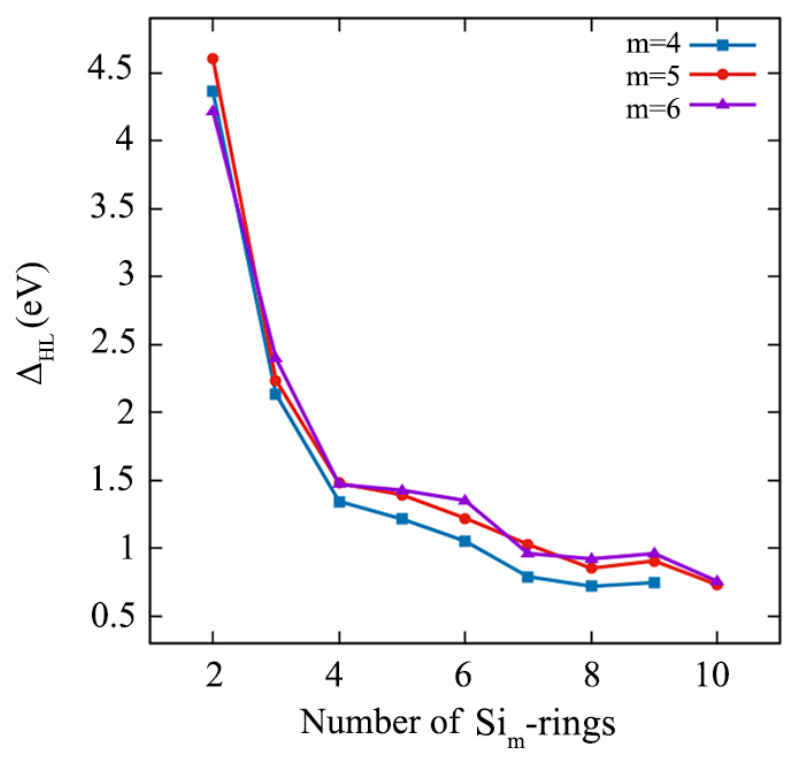

Fig. 3. HOMO-LUMO gaps $\Delta_{\mathrm{HL}}$ versus the number of $\mathrm{Si}_{m}$-rings $n$ obtained at the DFT/B3LYP/6-311G(d,p) level of theory $(m=4,5$, and 6). 
out to be thermodynamically unstable when the number of layers $n \geq 10$. Binding energies estimated for "infinite" pentaand hexasilaprismanes are equal to 3.16 and $3.15 \mathrm{eV} /$ atom, respectively. In contrast to binding energies, HOMO-LUMO gaps decrease as the effective length of polysilaprismane increases. In the bulk limit $(n \rightarrow \infty) \Delta_{\mathrm{HL}}$ is equal to 0.17 and $0.15 \mathrm{eV}$ for $[n, 5]$ - and $[n, 6]$ silaprismanes, respectively. These values indicate that the long silaprismanes can be referred to metals and can be successfully used in nanoelectronics as functional nanowires or basis for computational logic elements without any additional doping or mechanical stresses.

Acknowledgments. The presented study was performed with the financial support of the Russian Science Foundation (Grant No. 18-72-00183).

\section{References}

1. P.A. S. Autreto, S.B. Legoas, M.Z. S. Flores, D.S. Galvao. J. Chem. Phys. 133, 124513 (2010). DOI: $10.1063 / 1.3483237$

2. A. Poater, A. G. Saliner, R. Carbó-Dorca, J. Poater, M. Solà, L. Cavallo, A. P. Worth. J. Comput. Chem. 30, 275 (2009). DOI: $10.1002 /$ jcc. 21041

3. A. Poater, A. G. Saliner, L. Cavallo, M. Poch, M. Solà, A.P. Worth. Curr. Med. Chem. 19, 5219 (2012). DOI: $10.2174 / 092986712803530548$

4. K. Ohno, H. Tokoyama, H. Yamakado. Chem. Phys. Lett. 635, 180 (2015). DOI: 10.1016/j.cplett.2015.06.060

5. K.P. Katin, S.A. Shostachenko, A.I. Avkhadieva, M.M. Maslov. Adv. Phys. Chem. 2015, 506894 (2015). DOI: $10.1155 / 2015 / 506894$

6. E.A. Belenkov, V.A. Greshnyakov. Phys. Solid State. 55(8), 1754 (2013). DOI: 10.1134/S1063783413080039

7. E. A. Belenkov, V.A. Greshnyakov. New Carbon Materials 28(4), 273 (2013). DOI: 10.1016/S1872-5805(13)60081-5

8. J. A. Baimova, L. Kh. Rysaeva. J. Struct. Chem. 59(4), 884 (2018). DOI: 10.1134/S0022476618040200

9. M.I. Tingaev, E. A. Belenkov. J. Phys.: Conf. Ser. 917, 032013 (2017). DOI: 10.1088/1742-6596/917/3/032013

10. J.A. Baimova, L.Kh. Rysaeva, S.V. Dmitriev, D.S. Lisovenko, V.A. Gorodtsov, D.A. Indeitsev. Mat. Phys. Mech. 33, 1 (2017). DOI: 10.18720/MPM.3312017_1

11. L. Pavesi, R. Turan. Silicon Nanocrystals: Fundamentals, Synthesis, and Applications. Wiley-VCH Verlag GmbH\&Co, Weinheim, Germany (2010).

12. S. Yang, W. Li, B. Cao, H. Zeng, W. Cai, J. Phys. Chem. C. 115(43), 21056 (2011). DOI: 10.1021/jp2075836

13. L.Z. Zhao, W.C. Lu, W.S. Su, W. Qin, C.Z. Wang, K. M. Ho, Phys. Chem. Chem. Phys. 17(41), 27734 (2015). DOI: $10.1039 / \mathrm{C} 5 \mathrm{CP} 03856 \mathrm{~A}$

14. Y. Yong, X. Hao, C. Li, X. Li, T. Li, H. Cui, S. Lv. RSC Adv.
5(48), 38680 (2015). DOI: 10.1039/C5RA02081F

15. B.X. Li, J.H. Liu, S.C. Zhan. Eur. Phys. J. D. 32(1), 59 (2005). DOI: 10.1140/epjd/e2004-00173-4

16. M. B. Ferraro, J. Comput. Methods Sci. Eng. 7, 195 (2007).

17. D. Yao, G. Zhang, B. Li. Nano Lett. 8(12), 4557 (2008). DOI: $10.1021 / \mathrm{nl} 802807 \mathrm{t}$

18. Z. Wu, J. B. Neaton, J. C. Grossman. Nano Lett. 9(6), 2418 (2009). DOI: $10.1021 / \mathrm{nl} 9010854$

19. W. Zhigang, J.B. Neaton, J.C. Grossman. Phys. Rev. Lett. 100(24), $246904 \quad$ (2008). DOI: 10.1103/PhysRevLett.100.246804

20. Q. Zhang, W. Zhang, W. Wan, Y. Cui, E. Wang. Nano Lett. 10(9), 3243 (2010). DOI: 10.1021/n1904132v

21. M.C. Wingert, S. Kwon, M. Hu, D. Poulikakos, J. Xiang, R. Chen. Nano Lett. 15(4), 2605 (2015). DOI: 10.1021/acs.nanolett.5b00167

22. R. Epur, P. J. Hanumantha, M. K. Datta, D. Hong, B. Gattu, P. N. Kumta. J. Mater Chem. A. 3(20), 11117 (2015). DOI: $10.1039 / \mathrm{C} 5 \mathrm{TA00961H}$

23. H. Matsumoto, K. Higuchi, S. Kyushin, M. Goto, Angew. Chem.Int. Ed. Engl. 31, 1354 (1992). DOI: 10.1002/anie.199213541

24. A. Sekiguchi, T. Yatabe, C. Kabuto, H. Sakurai. J.Am. Chem. Soc. 115, 5853 (1993). DOI: $10.1021 / \mathrm{ja} 00066 \mathrm{a} 075$

25. C. Lee, W. Yang, R. G. Parr. Phys. Rev. B. 37, 785 (1988). DOI: 10.1103/PhysRevB.37.785

26. A.D. Becke, J. Chem. Phys. 98, 5648 (1993). DOI: $10.1063 / 1.464913$

27. R. Krishnan, J. S. Binkley, R. Seeger, J. A. Pople. J. Chem. Phys. 72(1), 650 (1980). DOI: 10.1063/1.438955

28. M. B. Javan. Phys. E. 67, 135 (2015). DOI: $10.1016 /$ j.physe.2014.11.008

29. D. L. Strout. J. Phys. Chem. A. 110(11), 4089 (2006). DOI: 0.1021/jp0563540

30. E. A. Belenkov, V.A. Greshnyakov. J. Mater. Sci. 50(23), 7627 (2015). DOI: 10.1007/s10853-015-9325-1

31. M. M. Maslov, K.P. Katin, Chem. Phys. Lett. 644, 280 (2016). DOI: 10.1016/j.cplett.2015.12.022

32. R. G. Parr, W. Yang. Density-Functional Theory of Atoms and Molecules. Oxford University Press, New York, USA (1989).

33. I.S. Ufimtsev, T. J. Martínez. J. Chem. Theory Comput. 5(10), 2619 (2009). DOI: 10.1021/ct9003004

34. A.V. Titov, I.S. Ufimtsev, N. Luehr, T.J. Martínez. J. Chem. Theory Comput. 9(1), 213 (2013). DOI: $10.1021 / \mathrm{ct} 300321 \mathrm{a}$

35. J. Kästner, J.M. Carr, T.W. Keal, W. Thiel, A. Wander, P. Sherwood. J. Phys. Chem. A. 113(43), 11856 (2009). DOI: $10.1021 /$ jp 9028968

36. T. P. M. Goumans, C. R. A. Catlow, W. A. Brown, J. Kästner and P. Sherwood. Phys. Chem. Chem. Phys. 11, 5431 (2009). DOI: 10.1039/B816905E 